\title{
Cardiovascular complications and acute ischemic stroke after the treatment with epinephrine in an anaphylactic patient
}

\author{
Ataman Köse*, Akif Yarkaç, Gülten Bozali, Seyran Bozkurt Babuş, Ersin Altınsoy \\ Department of Emergency Medicine, Faculty of Medicine, Mersin University, Mersin, Turkey
}

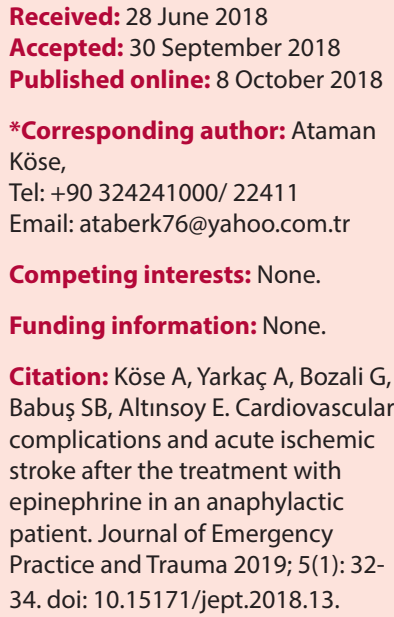

\begin{abstract}
Anaphylaxis is a serious clinical condition that can affect all ages and many systems (skin, gastrointestinal system, respiratory and cardiovascular systems). There are a few case reports of cardiac and cerebrovascular complications due to the anaphylaxis and/or the treatment with epinephrine. A 69-year-old female patient with known coronary artery disease (CAD), coronary artery bypass graft (CABG), and diabetes mellitus (DM) was admitted to the emergency department. She was brought to the emergency service with the complaints of shortness of breath, swelling of the tongue and lip, widespread itching and nausea after the use of amoxicillin-clavulonic acid due to soft tissue infection. The patient was considered as having anaphylaxis. $0.5 \mathrm{mg}$ intramuscular (IM) epinephrine was administered. Following the emergency electrocardiography (ECG), the rate was $140 / \mathrm{min}$ and the rhythm was evaluated as atrial fibrillation. We present a case of cardiovascular complications and acute ischemia stroke following intramuscular epinephrine administration with anaphylactic diagnosis in this study. As a result, anaphylaxis management is extremely important in elderly patients with preexisting cardiovascular conditions.

Keywords: Anaphylaxis, Epinephrine, Stroke
\end{abstract}

\section{Introduction}

Anaphylaxis is a serious clinical condition that can affect all ages and many systems (skin, gastrointestinal system, respiratory and cardiovascular systems). Anaphylaxis causes the release of vasoactive chemical agents from mast cells $(1,2)$. There are a few case reports of cardiac and cerebrovascular complications due to the anaphylaxis and/or the treatment with epinephrine. We present a case of cardiovascular complication and stroke following intramuscular (IM) adrenalin administration with anaphylactic diagnosis in this study.

\section{Case report}

A 69-year-old female patient with known coronary artery disease (CAD), coronary artery bypass graft (CABG), and diabetes mellitus (DM) was admitted to the emergency department. She was brought to the emergency service with the complaints of shortness of breath, swelling of the tongue and lip, widespread itching and nausea after the use of amoxicillin-clavulonic acid due to soft tissue infection. The vital signs of the patient included a blood pressure of $90 / 60$, pulse rate of $130 / \mathrm{min}$, respiratory rate of $30 / \mathrm{min}$, and $\mathrm{O}_{2}$ saturation with pulse oximeter of 90 . On physical examination, the patient had pale, sweaty, and chronotropic appearance, in addition, bilateral bronchospasm was present in the lungs. The tongue and lips were swollen and edema was apparent. On first electrocardiography (ECG), there was no acute ischemic change at normal sinus rhythm of $120 / \mathrm{min}$. The patient was considered as having anaphylaxis and she was monitored by opening the vein pathway. Intravenous (IV) fluid hydration and $0.5 \mathrm{mg}$ IM epinephrine were administered. Besides, oxygen therapy (3-4 L/dk) was initiated. The patient was given salbutamol inhaler, an ampule antihistamine (pheniramine 22.7 $\mathrm{mg}$ ) and corticosteroid IV $80 \mathrm{mg}$ (methylprednisolone). Consequently, her blood pressure improved. We reapplied $0.3 \mathrm{mg}$ of epinephrine IM for continuing tachypnea, abdominal pain and widespread itching. ECG monitoring in the emergency department showed a rate of $140 / \mathrm{min}$ and the rhythm was evaluated as atrial fibrillation. In D2D3-aVF, V5-V6 a nonspecific ST-T change was developed and the patient was consulted to cardiology department with the initial diagnosis of coronary vasospasm due to allergic reaction. No acute pathology was detected on 
echocardiography. No serious abnormal findings were found in patient's blood tests and cardiac enzymes were negative. In the follow-up of the patient, he developed speech impairment and weakness in the right upper extremity. On neurological examination, the patient had facial asymmetry and right upper extremity $1 / 5$ motor deficit. Within about 30 minutes, motor deficit gradually improved. In the emergency department, the motor deficit in the right upper extremity was $4 / 5$. Cerebral diffuse magnetic resonance imaging revealed a lesion in the left middle cerebral artery and posterior cerebral artery compatible with acute infarction (Figure 1). The patient was referred to the center for intensive care by the neurology department with the diagnosis of acute ischemic stroke.

\section{Discussion}

Anaphylaxis is life threatening medical emergency. Previously, brain damage have been described in the anaphylaxis and anaphylactic shock (3). Vasoactive mediators released by anaphylaxis (histamine, tumor necrosis factor-alpha, heparin and neutral proteases) are reported to contribute to blood-brain barrier damage, brain edema, prolonged extravasation and haemorrhage (4). Early application of epinephrine is extremely important to prevent anaphylactic side effects from progressing to life-threatening conditions. However, problems such as cerebral perfusion disorder and cerebral ischemia due to vasospasm have been reported especially due to intravenous use of adrenaline $(5,6)$. Evidence shows that arterial hypotension may also impair cerebral blood flow. In the study of Davidson et al, cerebral ischemia and cerebral damage following anaphylactic shock were reported not to be due to arterial hypotension but to be directly influenced by anaphylactic mediators on the cerebral artery system (7).

Similarly, the underlying mechanism of anaphylactic cardiac complications (acute coronary syndrome, myocardial infarction, arrhythmia, myocarditis, etc) is independent of the etiology (vasoactive mediators and catecholamine release, vasospasm and microvascular dysfunction). In addition, the use of epinephrine will increase the potential for the development of arrhythmias. The incidence of cardiovascular complications due to the use of IV epinephrine is especially higher. Therefore, the use of IM is highly recommended $(1,2)$.

Three factors may have contributed to the development of cardiac and cerebrovascular complications in our case. These include (a) anaphylaxis and vasochemical mediators released, (b) arterial hypotension due to anaphylaxis in the patient, and (c) sympathetic effect of epinephrine. It is difficult to analyze exactly what triggers these, but the emergence of these complications especially after epinephrine is more prominent than others. It is also important that the patient is old and has a previous

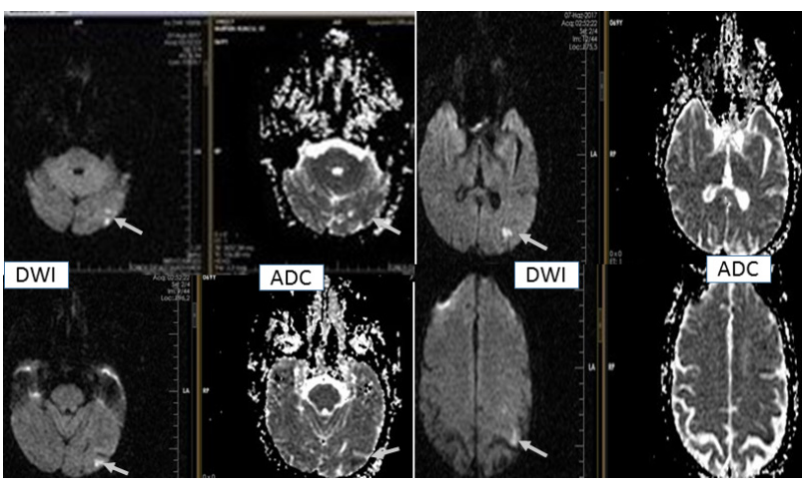

Figure 1. Cerebral diffuse magnetic resonance showed a lesion in the left middle cerebral artery and posterior cerebral artery that was compatible with acute infarction.

Abbreviations: DWI, diffusion-weighted imaging; ADC, Apparent diffusion coefficient.

cardiac disease history. The use of epinephrine in elderly patients with CAD as well as the concern about the potential cardiac effects are important and should be well considered against the risk of untreated anaphylaxis.

Therefore, anaphylaxis management is extremely important in elderly patients with preexisting cardiovascular conditions. Particular attention should be paid to the use of epinephrine and cardiac and cerebrovascular complications that may develop in the anaphylactic patients. Emergency physicians should have information about these outcomes.

\section{Ethical approval}

Not needed.

\section{Authors' contributions}

AY, GB, EA collected the clinical data. AK and SBB searched literature, wrote and clarified the manuscript. AK revised and edited the manuscript. All authors read and approved the final manuscript.

\section{References}

1. Tummala K, Maniyal VK, Chandrashekaran R, Mathew N, Ganeshwala G. Cardiac anaphylaxis: a case of acute ST-segment elevation myocardial infarction after IM epinephrine for anaphylactic shock. Am J Emerg Med 2013; 31(7): 1157.e1-3. doi: 10.1016/j.ajem.2013.03.025.

2. Mueller UR. Cardiovascular disease and anaphylaxis. Curr Opin Allergy Clin Immunol 2007; 7(4): 337-41. doi: $\quad 10.1097 /$ ACI.0b013e328259c328.

3. Ding YJ, Song H, Liu JH, Wang GH. Brain injury due to anaphylactic shock as a result of formocresol used during root canal treatment. Int Endod J 2013; 46(10): 999-1005. doi: 10.1111/iej.12092.

4. Lindsberg PJ, Strbian D, Karjalainen-Lindsberg ML. Mast cells as early responders in the regulation of acute blood-brain barrier changes after cerebral ischemia and hemorrhage. J Cereb Blood Flow Metab 2010; 30(4): 689-702. doi: $10.1038 / j \mathrm{cbfm} .2009 .282$. 
5. Ristagno G, Tang W, Huang L, Fymat A, Chang YT, Sun $S$, et al. Epinephrine reduces cerebral perfusion during cardiopulmonary resuscitation. Crit Care Med 2009; 37(4): 1408-15. doi: 10.1097/CCM.0b013e31819cedc9.

6. Saxena N, Sharma M. Cerebral infarction following carotid arterial injection of adrenaline. Can J Anaesth 2005; 52(1): 119. doi: 10.1007/bf03018600.

7. Davidson J, Zheng F, Tajima K, Barthel G, Alb I, Tabarna A, et al. Anaphylactic shock decreases cerebral blood flow more than what would be expected from severe arterial hypotension. Shock 2012; 38(4): 429-35. doi: 10.1097/SHK.0b013e3182690897. 